Article

\title{
Biological Validation of Novel Polysubstituted Pyrazole Candidates with in Vitro Anticancer Activities
}

\author{
Hoda H. Fahmy ${ }^{1}$, Nagy M. Khalifa ${ }^{1,2, *}$, Magda M. F. Ismail ${ }^{3}$, Hend M. El-Sahrawy ${ }^{3}$ \\ and Eman S. Nossier ${ }^{3}$ \\ 1 Department of Therapeutical Chemistry, Pharmaceutical and Drug Industries Division, \\ National Research Centre, Giza 12622, Egypt; hh_fahmy@yahoo.com \\ 2 Pharmaceutical Chemistry Department, Drug Exploration \& Development Chair (DEDC), \\ College of Pharmacy, King Saud University, Riyadh 11451, Saudi Arabia \\ 3 Department of Pharmaceutical Chemistry, Faculty of Pharmacy (Girls), Al-Azhar University, \\ Cairo 11754, Egypt; m.elalfy101@gmail.com (M.M.F.I.); hendelsehrawi@hotmail.com (H.M.E.); \\ emy28_s@hotmail.com (E.S.N.) \\ * Correspondence: nagykhalifa@hotmail.com or nkhalifa.c@ksu.edu.sa; Tel.: +966-14-677-343; \\ Fax: +966-14-676-220
}

Academic Editor: Maria Emília de Sousa

Received: 3 December 2015 ; Accepted: 19 February 2016 ; Published: 26 February 2016

\begin{abstract}
With the aim of developing novel antitumor scaffolds, a novel series of polysubstituted pyrazole derivatives linked to different nitrogenous heterocyclic ring systems at the C-4 position were synthesized through different chemical reactions and characterized by means of spectral and elemental analyses and their antiproliferative activity against 60 different human tumor cell lines was validated by the U.S. National Cancer Institute using a two stage process. The in vitro anticancer evaluation revealed that compound 9 showed increased potency toward most human tumor cell lines with $\mathrm{GI}_{50} \mathrm{MG}-\mathrm{MID}=3.59 \mu \mathrm{M}$, as compared to the standard drug sorafenib $\left(\mathrm{GI}_{50} \mathrm{MG}-\mathrm{MID}=1.90 \mu \mathrm{M}\right)$. At the same time, compounds $6 \mathbf{a}$ and 7 were selective against the HOP-92 cell line of non-small cell lung cancer with $\mathrm{GI}_{50} 1.65$ and $1.61 \mu \mathrm{M}$, respectively.
\end{abstract}

Keywords: 1,3,4-polysubstituted pyrazole derivatives; anticancer agents; synthesis

\section{Introduction}

The development of new antitumor agents is an important field of scientific activity, due to the toxic side effect problems of recent drugs. Many of the obtainable anticancer agents display unwanted side effects such as reduced bioavailability, toxicity and drug-resistance [1-5]. Incorporation of a pyrazole ring into different heteroaryl ring systems results in significant anticancer activities [6-9]. Different substituted pyrazole compounds have also been examined for their antiproliferative activities in vitro and antitumor activity in vivo, resulting in promising target products [10-12]. On the other hand, compounds containing pyrazole derivatives represent an advantageous choice for the synthesis of compounds with a broad spectrum of pharmacological activities, including anti-inflammatory [13], antibacterial, antifungal [14], inhibition of cyclooxygenase-2 [15], antiangiogenic [16], antipyretic [17], antihypertensive [18], antiplatelet [19], nitric oxide synthase (NOS) inhibitors [20] and anticancer activities [21]. Based on these observations and in continuation of our research on biologically active heterocycles [22-25], it was of interest to incorporate the 1,2,4-polysubstituted pyrazole ring system into different heteroaryl ring systems in one molecule in an attempt to obtain a new target anticancer agents. 


\section{Results and Discussion}

\subsection{Chemistry}

The reaction sequences outlined in Schemes 1 and 2 were used for the synthesis of the target compounds. Application of the Claisen Schmidt condensation on 2-acetylthiophene and 1-(3-chlorophenyl)-3-(4-methoxyphenyl)-1H-pyrazole-4-carboxaldehyde (1) in ethanolic sodium hydroxide solution according to literature methods [26,27] afforded (E)-3-(1-(3-chlorophenyl)-3-(4methoxyphenyl)-1H-pyrazol-4-yl)-1-(thiophen-2-yl)prop-2-en-1-one (2), which was used as starting material. Cyclocondensation of the $\alpha, \beta$-unsaturated ketone 2 with hydrazine hydrate in absolute ethanol or glacial acetic acid yielded the corresponding pyrazoline derivative 3 and $\mathrm{N}$-acetyl-pyrazoline derivative 4, respectively. On the other hand, heating of 2 with thiosemicarbazide in ethanolic $\mathrm{NaOH}$ gave 1-thiocarbamoyl pyrazole derivative 5 . In addition, condensation of compound $\mathbf{1}$ with ethyl cyanoacetate, or ethyl acetoacetate in the presence of guanidine hydrochloride gave 2-amino-5-cyano/acetyl-6-hydroxy-4-aryl pyrimidines 6a,b, respectively (Scheme 1).

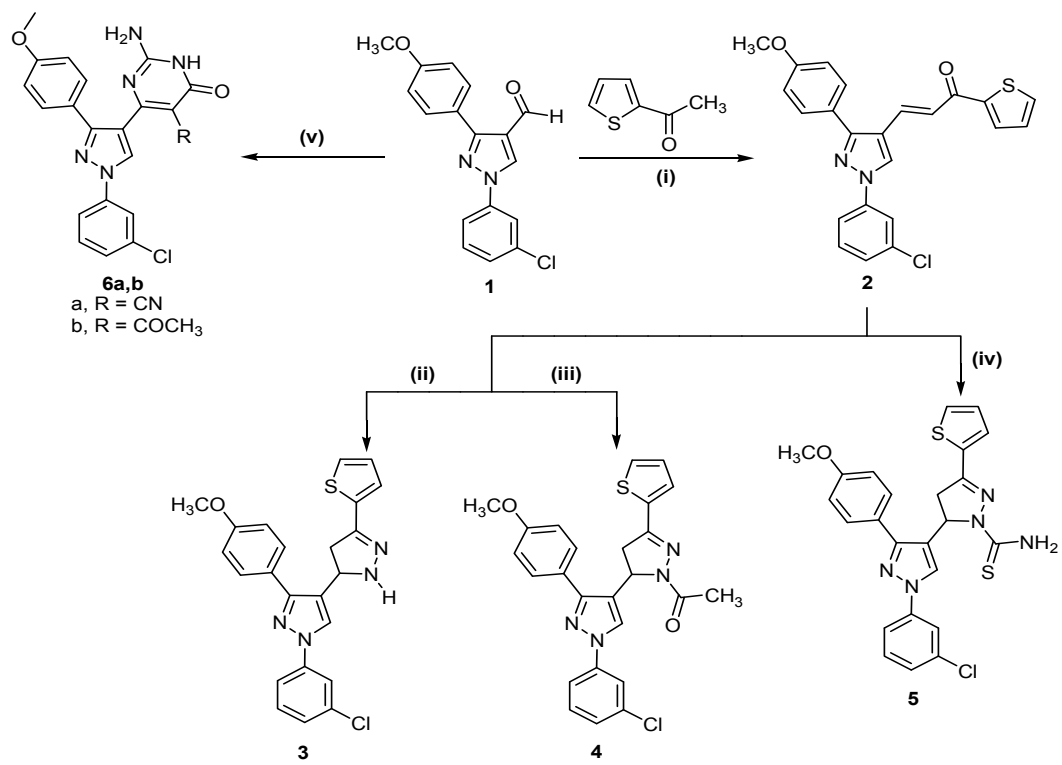

Reagents and Conditions: (i) ethanol/NaOH/rt./12 h, 87\%; (ii) $\mathrm{N}_{2} \mathrm{H}_{4} \cdot \mathrm{H}_{2} \mathrm{O} /$ ethanol/reflux/6 h, $57 \%$; (iii) $\mathrm{N}_{2} \mathrm{H}_{4} \cdot \mathrm{H}_{2} \mathrm{O} / \mathrm{AcOH} /$ reflux $/ 3 \mathrm{~h}, 65 \%$; (iv) $\mathrm{NH}_{2} \mathrm{NHCSNH}_{2} /$ ethanol/NaOH$/$ reflux $/ 5 \mathrm{~h}, 61 \%$; (v) NC-CH2COOEt or $\mathrm{CH}_{3} \mathrm{COCH}_{2} \mathrm{COOEt}, \mathrm{H}_{2} \mathrm{~N}-\mathrm{C}(\mathrm{NH})-\mathrm{NH}_{2} \cdot \mathrm{HCl} /$ ethanol$/ \mathrm{NaOH} /$ reflux $3 \mathrm{~h}, 64-68 \%$.

Scheme 1. Synthetic route for trisubstituted pyrazole compounds 2-6.

Finally, $\alpha, \beta$-unsaturated ketone 2 was reacted with hydroxylamine hydrochloride in refluxing ethanol in the presence of sodium hydroxide as alkaline medium to afford the corresponding isoxazoline 7 . Treatment of $\mathbf{2}$ with guanidine sulfate in ethanolic sodium hydroxide gave 2-aminopyrimidine derivative $\mathbf{8}$, which was reacted with thiourea in the presence of sodium hydroxide to give the corresponding pyrimidine-2-thione derivative 9 (Scheme 2).

\subsection{In Vitro Anticancer Screening}

The target compounds were selected by the U.S. National Cancer Institute (NCI), for anticancer activity screening. The screening is a two-stage process, beginning with the evaluation at a single dose $(10 \mu \mathrm{M})$ and the compounds which display significant growth inhibition are then evaluated at five concentration levels. The first screening, where the selected compounds are evaluated at a single dose $(10 \mu \mathrm{M})$ and the culture is incubated for $48 \mathrm{~h}$, utilizes 60 different human tumor cell lines, representing leukemia, melanoma and cancers of lung, colon, central nervous system (CNS), ovary, kidney, prostate as well as breast. 


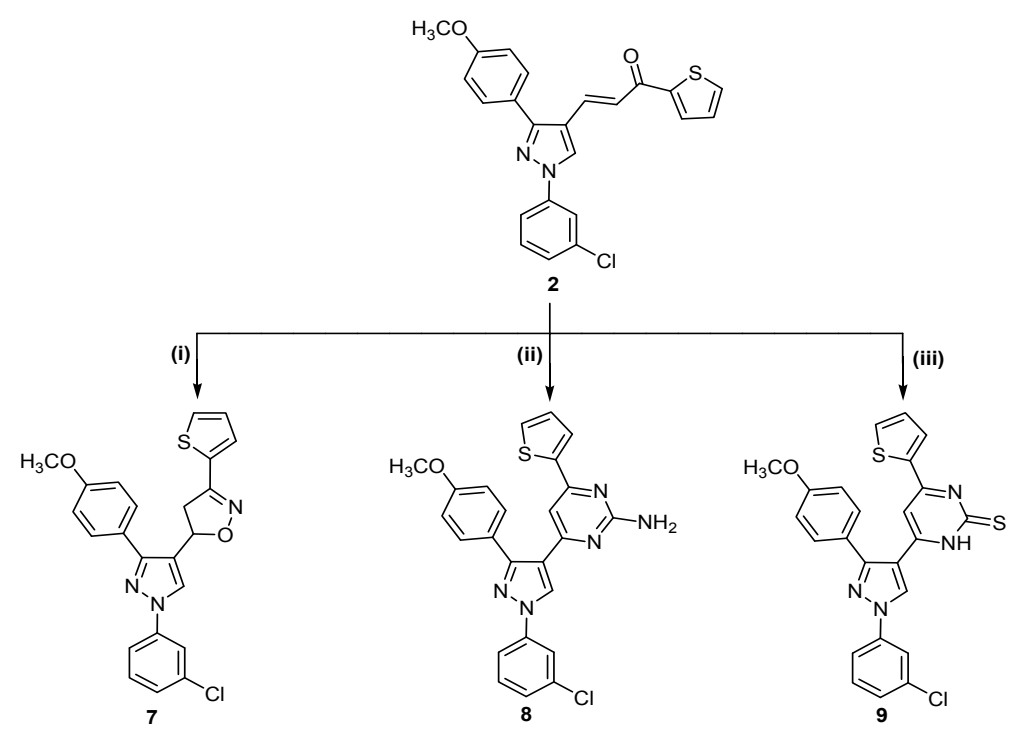

Reagents and Conditions: (i) $\mathrm{NH}_{2} \mathrm{OH} \cdot \mathrm{HCl} /$ ethanol/NaOH/reflux/3 h, 73\%; (ii) $\mathrm{H}_{2} \mathrm{~N}-\mathrm{C}(\mathrm{NH})-\mathrm{NH}_{2} \cdot \mathrm{H}_{2} \mathrm{SO}_{4}$ / ethanol/NaOH reflux/5 h, 67\%; (iii) $\mathrm{NH}_{2} \mathrm{CSNH}_{2} /$ ethanol/NaOH/reflux $/ 6 \mathrm{~h}, 76 \%$.

Scheme 2. Synthetic route for trisubstituted pyrazole compounds 7-9.

The percentages of growth of the tested compounds against the full 60-cell line panel are listed in Table 1. The one dose mean graphs of the selected compounds revealed that compounds $6 \mathbf{a}, 7$ and 9 showed increased potency against most human cancer cell lines, so these compounds were selected for further evaluation at five dose concentration levels $(0.01-100 \mu \mathrm{M})$.Regarding sensitivity against individual cell lines, compound 9 showed potent anticancer activity against all human cancer cell lines with $\mathrm{GI}_{50} 1.9-5.50 \mu \mathrm{M}$. It had the highest selectivity against the non-small cell lung cancer cell line EKVX, with $\mathrm{GI}_{50} 1.9 \mu \mathrm{M}$. At the same time, compounds $6 \mathrm{a}$ and 7 showed the highest activity against the cell line HOP-92 belonging to the non-small cell lung cancer class with $\mathrm{GI}_{50} 1.7$ and $1.6 \mu \mathrm{M}$, respectively, and against the renal cancer cell line $\mathrm{A} 498$ with $\mathrm{GI}_{50} 1.47$ and $1.81 \mu \mathrm{M}$, respectively.

Examination of all the ligand structural modifications performed at the 4-position of 1,3,4-trisubstituted pyrazole scaffold to establish the structure activity relationships with anticancer acitivity shows the following results: first, introduction of the pyrimidine-2(1H)-thione moiety in the 4-position of the pyrazole moiety in compound 9 enhanced the potency towards most cancer cell lines. It has $\mathrm{GI}_{50} \mathrm{MG}-\mathrm{MID}=3.6 \mu \mathrm{M}$ against all subpanel tumor cell lines, comparable to that of sorafenib $\left(\mathrm{GI}_{50} \mathrm{MG}-\mathrm{MID}=1.90 \mu \mathrm{M}\right.$. Concerning the pyrazolyl derivative $6 \mathbf{a}$, it was observed that the activity was enhanced in 2-amino-6-oxopyrimidine-5-carbonitrile, which suggests that the polar 6-aminopyrimidine moiety has a role in enhancing anticancer activity $\left(\mathrm{GI}_{50} \mathrm{MG}-\mathrm{MID}=5.70 \mu \mathrm{M}\right)$, but less so than the 2-mercaptopyrimidine group. Finally, introduction of a 1-carbothioamide to a pyrazole or isoxazoline group substituted on the pyrazole greatly reduces the activity and these compounds showed the least potent activity. The results are presented in Tables 1-6.

Table 1. The mean growth percent of compounds $2,3,4,6 a, 6 b, 7,8$ and 9.

\begin{tabular}{ccc}
\hline Cpd. No. & NSC No. & Mean Growth Percent \\
\hline $\mathbf{2}$ & $762925 / 1$ & 95.56 \\
$\mathbf{3}$ & $763580 / 1$ & 102.40 \\
$\mathbf{4}$ & $763581 / 1$ & 98.43 \\
$\mathbf{6 a}$ & $762930 / 1$ & 67.27 \\
$\mathbf{6 b}$ & $762931 / 1$ & 76.45 \\
$\mathbf{7}$ & $762927 / 1$ & 47.83 \\
$\mathbf{8}$ & $762928 / 1$ & 99.26 \\
$\mathbf{9}$ & $762929 / 1$ & 49.53 \\
\hline
\end{tabular}


Table 2. $\mathrm{GI}_{50}(\mu \mathrm{M})$ of five-dose screening results of compounds $\mathbf{6 a}, \mathbf{7}$ and $\mathbf{9 .}$

\begin{tabular}{|c|c|c|c|}
\hline \multicolumn{4}{|c|}{ Subpanel Cell Lines } \\
\hline \multicolumn{4}{|c|}{$\mathrm{GI}_{50}$} \\
\hline Cell lines & $6 a$ & 7 & 9 \\
\hline \multicolumn{4}{|c|}{ Leukemia } \\
\hline CCRF-CEM & 3.65 & 3.84 & 2.80 \\
\hline HL-60(TB) & 3.96 & 4.73 & 3.49 \\
\hline K-562 & 3.45 & 3.43 & 2.91 \\
\hline MOLT-4 & 4.80 & 2.96 & 3.03 \\
\hline RPMI-8226 & 5.63 & 5.33 & 2.42 \\
\hline SR & 3.73 & 3.03 & 4.00 \\
\hline \multicolumn{4}{|c|}{ Non-Small Cell Lung Cancer } \\
\hline A549/ATCC & 5.13 & 3.56 & 2.29 \\
\hline EKVX & 3.29 & 2.07 & 1.93 \\
\hline HOP-62 & 10.1 & 13.90 & 7.14 \\
\hline HOP-92 & 1.65 & 1.61 & 2.20 \\
\hline NCI-H226 & 6.23 & 4.11 & 3.16 \\
\hline NCI-H23 & 5.44 & 3.82 & 3.43 \\
\hline NCI-H460 & 6.00 & 3.42 & 3.01 \\
\hline NCI-H522 & 3.11 & 4.27 & 2.54 \\
\hline \multicolumn{4}{|l|}{ NCI-H322M } \\
\hline \multicolumn{4}{|c|}{ Colon Cancer } \\
\hline COLO 205 & 7.65 & 3.21 & 2.35 \\
\hline HCC-2998 & 5.87 & 3.47 & 4.03 \\
\hline HCT-116 & 4.35 & 3.03 & 2.38 \\
\hline HCT-15 & 5.07 & 4.48 & 3.69 \\
\hline HТ29 & 4.29 & 3.85 & 3.82 \\
\hline KM12 & 3.84 & 2.98 & 3.67 \\
\hline SW-620 & 4.38 & 4.35 & 3.79 \\
\hline \multicolumn{4}{|c|}{ CNS Cancer } \\
\hline SF-268 & 5.86 & 4.94 & 4.52 \\
\hline SF-295 & 2.36 & 2.70 & 2.07 \\
\hline SF-539 & 6.59 & 7.47 & 4.70 \\
\hline SNB-19 & 4.58 & 6.08 & 4.93 \\
\hline SNB-75 & 2.88 & 3.52 & 3.49 \\
\hline U251 & 3.66 & 3.61 & 2.60 \\
\hline \multicolumn{4}{|c|}{ Melanoma } \\
\hline LOX IMVI & 4.02 & 3.04 & 3.56 \\
\hline MALME-3M & 5.83 & 2.60 & 3.49 \\
\hline M14 & 3.25 & 3.30 & 2.81 \\
\hline MDA-MB-435 & 2.16 & 3.26 & 3.13 \\
\hline SK-MEL-2 & 3.94 & 3.34 & 2.87 \\
\hline SK-MEL-28 & 4.98 & 4.93 & 3.93 \\
\hline SK-MEL-5 & 4.07 & 4.74 & 2.93 \\
\hline UACC-257 & 5.69 & 3.07 & 3.06 \\
\hline UACC-62 & 3.33 & 3.55 & 2.78 \\
\hline \multicolumn{4}{|c|}{ Ovarian Cancer } \\
\hline IGROV1 & 3.01 & 4 & 3.61 \\
\hline OVCAR-3 & 3.38 & 5.07 & 3.04 \\
\hline OVCAR-4 & 7.71 & 4.48 & 3.47 \\
\hline OVCAR-5 & 14.60 & 7.63 & 6.38 \\
\hline OVCAR-8 & 10.10 & 4.04 & 3.15 \\
\hline NCI/ADR-RES & 3.33 & 4.01 & 4.77 \\
\hline SK-OV-3 & 6.56 & 8.89 & 5.91 \\
\hline
\end{tabular}


Table 2. Cont.

\begin{tabular}{cccc}
\hline \multicolumn{5}{c}{ Renal Cancer } \\
\hline 786-0 & 4.65 & 3.39 & 4.18 \\
A498 & 1.47 & 1.81 & 3.01 \\
ACHN & 9.92 & 4.39 & 4.05 \\
CAKI-1 & 2.52 & 2.05 & 3.03 \\
RXF 393 & 4.51 & 3.49 & 3.76 \\
SN12C & 6.41 & 3.48 & 3.92 \\
TK-10 & 13.50 & 5.21 & 4.55 \\
UO-31 & 2.94 & 2.56 & 2.82 \\
\multicolumn{4}{c}{ Prostate Cancer } \\
PC-3 & 6.30 & 3.2 & 3.09 \\
DU-145 & 17.9 & 7.08 & 5.49 \\
\hline \multicolumn{3}{c}{ Breast Cancer } \\
MCF7 & 3.54 & 3.53 & 3.02 \\
MDA-MB-231/ATCC & 3.39 & 3.08 & 3.52 \\
HS 578T & 3.55 & 4.42 & 4.26 \\
BT-549 & 3.55 & 4.99 & 3.18 \\
T-47D & 6.05 & 3.86 & 4.16 \\
MDA-MB-468 & 2.12 & 2.68 & 2.57
\end{tabular}

$\mathrm{GI}_{50}$ : (growth inhibitory activity) the drug concentration that reduces cellular growth by $50 \%$.

Table 3. TGI ( $\mu \mathrm{M})$ of five-dose screening results of compounds $\mathbf{6 a}, \mathbf{7}$ and $\mathbf{9 .}$

\begin{tabular}{|c|c|c|c|}
\hline \multicolumn{4}{|c|}{ Subpanel Cell Lines } \\
\hline \multicolumn{4}{|c|}{ TGI } \\
\hline Cell lines & $6 a$ & 7 & 9 \\
\hline \multicolumn{4}{|c|}{ Leukemia } \\
\hline CCRF-CEM & 33.80 & $>100$ & $>100$ \\
\hline HL-60(TB) & 14.20 & 38.30 & 13.20 \\
\hline K-562 & $>100$ & $>100$ & $>100$ \\
\hline MOLT-4 & 39.80 & 19.50 & 25.20 \\
\hline RPMI-8226 & 55.50 & 61.90 & 10.90 \\
\hline SR & 21.20 & 12.10 & 31.90 \\
\hline \multicolumn{4}{|c|}{ Non-Small Cell Lung Cancer } \\
\hline A549/ATCC & 50.80 & 24.50 & 16.50 \\
\hline EKVX & 35.40 & 13.50 & 21.70 \\
\hline HOP-62 & 26.20 & 28.10 & 22.10 \\
\hline HOP-92 & 12.20 & 8.13 & 10.80 \\
\hline NCI-H226 & 70.300 & 30.30 & 54.40 \\
\hline NCI-H23 & 46.20 & 20.80 & 16.20 \\
\hline NCI-H460 & 27.70 & 16.60 & 11.00 \\
\hline NCI-H522 & 9.80 & 18.40 & 8.86 \\
\hline \multicolumn{4}{|l|}{ NCI-H322M } \\
\hline COLO 205 & 20.90 & 7.24 & 5.59 \\
\hline HCC-2998 & 24.90 & 14.60 & 16.40 \\
\hline HCT-116 & 16.20 & 12.70 & 11.20 \\
\hline HCT-15 & $>100$ & 32.90 & $>100$ \\
\hline HT29 & 20.00 & 15.10 & 13.00 \\
\hline KM12 & 15.20 & 11.10 & 14.40 \\
\hline SW-620 & 34.20 & 22.60 & 22.30 \\
\hline
\end{tabular}


Table 3. Cont.

\begin{tabular}{|c|c|c|c|}
\hline \multicolumn{4}{|c|}{ CNS Cancer } \\
\hline SF-268 & 35.4 & 47.10 & 73.60 \\
\hline SF-295 & 9.22 & 13.20 & 7.09 \\
\hline SF-539 & 32.2 & 36.60 & 19.70 \\
\hline SNB-19 & 100 & $>100$ & $>100$ \\
\hline SNB-75 & 21.1 & 34.70 & 25.60 \\
\hline U251 & 18.8 & 15.40 & 12.40 \\
\hline \multicolumn{4}{|c|}{ Melanoma } \\
\hline LOX IMVI & 20.10 & 10.90 & 13.80 \\
\hline MALME-3M & 40.60 & 10.60 & 15.80 \\
\hline M14 & 34.00 & 17.10 & 14.00 \\
\hline MDA-MB-435 & 5.48 & 17.30 & 19.00 \\
\hline SK-MEL-2 & 15.80 & 10.90 & 6.90 \\
\hline SK-MEL-28 & 27.90 & 17.80 & 15.70 \\
\hline SK-MEL-5 & 15.90 & 17.30 & 10.90 \\
\hline UACC-257 & 47.30 & 15.10 & 18.00 \\
\hline UACC-62 & 15.40 & 20.00 & 19.20 \\
\hline \multicolumn{4}{|c|}{ Ovarian Cancer } \\
\hline IGROV1 & 18.10 & 16.20 & 15.30 \\
\hline OVCAR-3 & 10.00 & 25.00 & 9.42 \\
\hline OVCAR-4 & 61.90 & 36.40 & $>100$ \\
\hline OVCAR-5 & 51.40 & 74.10 & $>100$ \\
\hline OVCAR-8 & 60.30 & 36.00 & $>100$ \\
\hline NCI/ADR-RES & 15.90 & 38.10 & 41.80 \\
\hline SK-OV-3 & 30.70 & 44.10 & 36.80 \\
\hline \multicolumn{4}{|c|}{ Renal Cancer } \\
\hline $786-0$ & 33.50 & $>100$ & $>100$ \\
\hline A498 & 6.75 & 14.60 & 17.00 \\
\hline $\mathrm{ACHN}$ & $>100$ & $>100$ & $>100$ \\
\hline CAKI-1 & 15.30 & 6.02 & 8.68 \\
\hline RXF 393 & 22.40 & 20.10 & 19.50 \\
\hline SN12C & $>100$ & 18.70 & $>100$ \\
\hline TK-10 & 53.90 & 43.80 & 32.80 \\
\hline UO-31 & 47.80 & 11.80 & 26.50 \\
\hline \multicolumn{4}{|c|}{ Prostate Cancer } \\
\hline PC-3 & $>100$ & 36.90 & $>100$ \\
\hline DU-145 & 70.10 & 35.80 & 72.50 \\
\hline \multicolumn{4}{|c|}{ Breast Cancer } \\
\hline MCF7 & 28.50 & 16.90 & 33.40 \\
\hline MDA-MB-231/ATCC & 53.30 & 16.90 & 26.00 \\
\hline HS $578 \mathrm{~T}$ & 40.30 & 45.50 & $>100$ \\
\hline BT-549 & 22.40 & 21.30 & 15.90 \\
\hline T-47D & 32.20 & 25.30 & 34.60 \\
\hline MDA-MB-468 & 6.43 & 12.30 & 9.91 \\
\hline
\end{tabular}

TGI: the drug concentration required for total growth inhibition. 
Table 4. $\mathrm{LC}_{50}(\mu \mathrm{M})$ of five-dose screening results of compounds $6 \mathrm{a}, 7$ and 9.

\begin{tabular}{|c|c|c|c|}
\hline \multicolumn{4}{|c|}{ Subpanel Cell Lines } \\
\hline \multicolumn{4}{|c|}{$\mathrm{LC}_{50}$} \\
\hline Cell lines & $6 a$ & 7 & 9 \\
\hline \multicolumn{4}{|c|}{ Leukemia } \\
\hline CCRF-CEM & $>100$ & $>100$ & $>100$ \\
\hline HL-60(TB) & $>100$ & $>100$ & $>100$ \\
\hline K-562 & $>100$ & $>100$ & $>100$ \\
\hline MOLT-4 & $>100$ & $>100$ & $>100$ \\
\hline RPMI-8226 & $>100$ & $>100$ & $>100$ \\
\hline SR & $>100$ & $>100$ & $>100$ \\
\hline \multicolumn{4}{|c|}{ Non-Small Cell Lung Cancer } \\
\hline A549/ATCC & $>100$ & $>100$ & $>100$ \\
\hline EKVX & $>100$ & $>100$ & $>100$ \\
\hline HOP-62 & 67.80 & 56.90 & 59.80 \\
\hline HOP-92 & $>100$ & $>100$ & 83.20 \\
\hline NCI-H226 & $>100$ & $>100$ & $>100$ \\
\hline NCI-H23 & $>100$ & $>100$ & $>100$ \\
\hline NCI-H460 & $>100$ & $>100$ & 52.00 \\
\hline NCI-H522 & $>100$ & 93.50 & $>100$ \\
\hline \multicolumn{4}{|l|}{ NCI-H322M } \\
\hline \multicolumn{4}{|c|}{ Colon Cancer } \\
\hline COLO 205 & $>50$ & 24.70 & 40.00 \\
\hline HCC-2998 & $>100$ & 64.70 & 96.00 \\
\hline НCT-116 & 46.00 & 40.60 & 33.80 \\
\hline HCT-15 & $>100$ & $>100$ & $>100$ \\
\hline HT29 & $>100$ & $>100$ & 46.90 \\
\hline KM12 & 49.70 & 84.60 & 49.30 \\
\hline SW-620 & $>100$ & $>100$ & $>100$ \\
\hline \multicolumn{4}{|c|}{ CNS Cancer } \\
\hline SF-268 & $>100$ & $>100$ & $>100$ \\
\hline SF-295 & 84.70 & $>100$ & 61.30 \\
\hline SF-539 & $>100$ & $>100$ & 82.30 \\
\hline SNB-19 & $>100$ & $>100$ & $>100$ \\
\hline SNB-75 & $>100$ & $>100$ & $>100$ \\
\hline U251 & 82.80 & 49.50 & 38.30 \\
\hline \multicolumn{4}{|c|}{ Melanoma } \\
\hline LOX IMVI & 74.10 & 54.90 & 45.20 \\
\hline MALME-3M & $>100$ & 60.00 & 97.20 \\
\hline M14 & $>100$ & 84.10 & 90.10 \\
\hline MDA-MB-435 & $>100$ & $>100$ & $>100$ \\
\hline SK-MEL-2 & 99.10 & 66.80 & 35.60 \\
\hline SK-MEL-28 & $>100$ & 52.40 & 58.80 \\
\hline SK-MEL-5 & 51.30 & 47.50 & 41.90 \\
\hline UACC-257 & $>100$ & $>100$ & $>100$ \\
\hline UACC-62 & 53.30 & 65.70 & 71.60 \\
\hline \multicolumn{4}{|c|}{ Ovarian Cancer } \\
\hline IGROV1 & $>100$ & 71.20 & 73.80 \\
\hline OVCAR-3 & 60.30 & $>100$ & 35.90 \\
\hline OVCAR-4 & $>100$ & $>100$ & $>100$ \\
\hline OVCAR-5 & $>100$ & 100 & $>100$ \\
\hline OVCAR-8 & $>100$ & $>100$ & $>100$ \\
\hline NCI/ADR-RES & $>100$ & $>100$ & $>100$ \\
\hline SK-OV-3 & $>100$ & $>100$ & $>100$ \\
\hline
\end{tabular}


Table 4. Cont.

\begin{tabular}{cccc}
\hline \multicolumn{5}{c}{ Renal Cancer } \\
\hline 786-0 & $>100$ & $>100$ & $>100$ \\
A498 & $>100$ & 45.20 & 73.20 \\
ACHN & $>100$ & $>100$ & $>100$ \\
CAKI-1 & $>100$ & 75.90 & $>100$ \\
RXF 393 & $>100$ & $>100$ & $>100$ \\
SN12C & $>100$ & $>100$ & $>100$ \\
TK-10 & $>100$ & $>100$ & $>100$ \\
UO-31 & $>100$ & $>100$ & $>100$ \\
\hline \multicolumn{5}{c}{ Prostate Cancer } \\
PC-3 & $>100$ & $>100$ & $>100$ \\
DU-145 & $>100$ & $>100$ & $>100$ \\
\hline \multicolumn{5}{c}{ Breast Cancer } \\
MCF7 & $>100$ & 83.60 & $>100$ \\
MDA-MB-231/ATCC & $>100$ & $>100$ & $>100$ \\
HS 578T & $>100$ & $>100$ & $>100$ \\
BT-549 & $>100$ & 66.30 & $>100$ \\
T-47D & $>100$ & $>100$ & $>100$ \\
MDA-MB-468 & $>100$ & $>100$ & $>100$ \\
\hline
\end{tabular}

$\mathrm{LC}_{50}$ : the drug concentration required for killing $50 \%$ of cells.

Table 5. Median growth inhibitory concentrations $\left(\mathrm{GI}_{50}, \mu \mathrm{M}\right)$ of in vitro subpanel tumor cell lines and $\mathrm{GI}_{50}(\mu \mathrm{M})$ full panel mean-graph mid-points (MG-MID) of compounds 6a, 7 and 9 in comparison with sorafenib.

\begin{tabular}{|c|c|c|c|c|c|c|c|c|c|c|}
\hline \multicolumn{11}{|c|}{ Subpanel Tumor Cell Lines } \\
\hline Cpd. No. & Leukemia & Lung & Colon & CNS & Melanoma & Ovarian & Renal & Prostate & Breast & GI $_{50}$ MG-MID \\
\hline $6 a$ & 4.2 & 5.1 & 5.1 & 4.3 & 4.1 & 7.0 & 5.7 & 12.1 & 3.7 & 5.7 \\
\hline 9 & 3.1 & 3.2 & 3.4 & 3.7 & 3.2 & 4.3 & 3.7 & 4.3 & 3.5 & 3.6 \\
\hline Sorafenib & & & & & & & & & & 1.9 \\
\hline
\end{tabular}

Table 6. Selectivity ratios for compounds 6a, 7 and 9 towards the nine tumor cell lines.

\begin{tabular}{cccccccccc}
\hline \multicolumn{10}{c}{ Subpanel Tumor Cell Lines } \\
\hline Cpd. No. & Leukemia & Lung & Colon & CNS & Melanoma & Ovarian & Renal & Prostate & Breast \\
\hline 6a & 1.4 & 1.1 & 1.1 & 1.3 & 1.4 & 0.8 & 1.0 & 0.5 & 1.5 \\
$\mathbf{7}$ & 1.1 & 0.9 & 1.2 & 0.9 & 1.2 & 0.8 & 1.3 & 0.8 & 1.1 \\
$\mathbf{9}$ & 1.2 & 1.1 & 1.1 & 1.0 & 1.1 & 0.8 & 1.0 & 0.8 & 1.0 \\
\hline
\end{tabular}

\section{Experimental Section}

\subsection{General Information}

Melting points were measured in open capillary tubes using a Griffin apparatus and are uncorrected. Structures of compounds were confirmed by routine spectrometric analysis. Elemental analyses were carried results were within $\pm 0.4 \%$ of the theoretical values. Infrared spectra were recorded on a $435 \mathrm{IR}$ spectrophotometer (Shimadzu Bruker, Tokyo, Japan) using KBr discs. ${ }^{1} \mathrm{H}-\mathrm{NMR}$ and ${ }^{13} \mathrm{C}$-NMR spectra were obtained on a Gemini $500 \mathrm{MHz}$ spectrophotometer (Varian, Polo Alto, Ca, USA) or on a Bruker $500 \mathrm{MHz}$ spectrophotometer, and measured in $\delta$ scale using TMS as an internal standard. Mass Spectra were recorded on a 5988 spectrometer (Hewlett Packard, California, USA). Analytical thin layer chromatography (TLC) was performed using silica gel aluminum sheets, $60 \mathrm{~F}_{254}$ 
(E. Merck, Darmstadt, Germany) for the progress of reactions and visualization with ultraviolet light (UV) at 365 and $254 \mathrm{~nm}$.

\subsection{3-(1-(3-Chlorophenyl)-3-(4-methoxyphenyl)-1H-pyrazol-4-yl)-1-(thiophen-2-yl)prop-2-en-1-one (2)}

A mixture of carbaldehyde derivative $1(0.01 \mathrm{~mol})$ and 2-acetylthiophene $(0.01 \mathrm{~mol})$ in of $30 \%$ ethanolic solution of $\mathrm{NaOH}(40 \mathrm{~mL})$ was stirred for $12 \mathrm{~h}$ at room temperature. The progress of reaction was monitored by TLC. After completion, the reaction mixture was poured into acidified ice cold water of $\mathrm{pH} 2$. The precipitated solid was filtered, washed with water and recrystallized to afford compound 2 in 87\% yield; m.p. $144-146^{\circ} \mathrm{C}(\mathrm{EtOH})$; IR (KBr) v: $3079(\mathrm{CH}-\mathrm{Ar}), 1641(\mathrm{C}=\mathrm{O}), 1600(\mathrm{C}=\mathrm{C}) \mathrm{cm}^{-1}$; ${ }^{1} \mathrm{H}-\mathrm{NMR}$ (DMSO- $\left.d_{6}\right): \delta 3.81\left(\mathrm{~s},{ }^{3} \mathrm{H}, \mathrm{OCH}_{3}\right) ; 7.00-7.98\left(\mathrm{~m},{ }^{12} \mathrm{H}, \mathrm{ArH}+\mathrm{CH}=\mathrm{CH}\right), 8.73\left(\mathrm{~s},{ }^{1} \mathrm{H}, \mathrm{CH}\right.$ of pyrazole) ppm; ${ }^{13} \mathrm{C}-\mathrm{NMR}\left(\mathrm{DMSO}-d_{6}\right): \delta 55.60,114.34,116.62,117.75,125.11,125.95,127.67,129.08$, 129.81, 131.74, 133.85, 134.47, 135.44, 141.08, 144.32, 151.54, 159.58, 191.96 ppm; MS (EI, $70 \mathrm{eV}): \mathrm{m} / z$ (\%): 420 (11) [M] ${ }^{+}$; Anal. Calcd for $\mathrm{C}_{23} \mathrm{H}_{17} \mathrm{ClN}_{2} \mathrm{O}_{2} \mathrm{~S}$ (420.91): C, 65.63; H, 4.07; N, 6.66; Found: C, 65.59; H, $4.16 ; \mathrm{N}, 6.72$.

\subsection{1-(3-Chlorophenyl)-4-(4,5-dihydro-3-(thiophen-2-yl)-1H-pyrazol-5-yl)-3-(4-methoxyphenyl)-1H-pyrazole (3)}

To a solution of compound $2(0.01 \mathrm{~mol})$ in ethanol $(30 \mathrm{~mL})$ containing a catalytic amount of glacial acetic acid, a solution of hydrazine hydrate $(98 \%, 0.5 \mathrm{~mL})$ was added and the mixture was refluxed for $6 \mathrm{~h}$. The reaction mixture was cooled to room temperature and the precipitated solid was filtered, dried and recrystallization provided compound 3 in $57 \%$ yield; m.p. $175-178^{\circ} \mathrm{C}(\mathrm{EtOH})$; IR (KBr) v: 3177 $(\mathrm{NH}), 1590(\mathrm{C}=\mathrm{C}) \mathrm{cm}^{-1},{ }^{1} \mathrm{H}-\mathrm{NMR}\left(\mathrm{DMSO}_{6}\right): \delta 2.99\left(\mathrm{dd},{ }^{1} \mathrm{H}, \mathrm{CH}\right), 3.70\left(\mathrm{~s},{ }^{3} \mathrm{H}, \mathrm{OCH}_{3}\right), 3.84\left(\mathrm{dd},{ }^{1} \mathrm{H}\right.$, $\mathrm{CH}), 5.42\left(\mathrm{dd},{ }^{1} \mathrm{H}, \mathrm{CH}\right), 6.39-7.77\left(\mathrm{~m},{ }^{11} \mathrm{H}, \mathrm{Ar}-\mathrm{H}\right), 8.91\left(\mathrm{~s},{ }^{1} \mathrm{H}, \mathrm{CH}\right.$ of pyrazole $), 11.52\left(\mathrm{~s},{ }^{1} \mathrm{H}, \mathrm{NH} \mathrm{D}_{2} \mathrm{O}\right.$ exchangeable) ppm; ${ }^{13} \mathrm{C}-\mathrm{NMR}$ (DMSO- $d_{6}$ ): $\delta 42.10,55.61,60.74,114.22,116.78,117.76,125.18,125.90$, 126.17, 127.38, 128.67, 129.65, 130.87, 131.26, 134.53, 137.55, 140.98, 151.24, 159.73, 158.76, 161.48 ppm; MS (EI, $70 \mathrm{eV}): m / z(\%): 434(18)[\mathrm{M}]^{+}$; Anal. Calcd for $\mathrm{C}_{23} \mathrm{H}_{19} \mathrm{ClN}_{4} \mathrm{OS}$ (434.94): C, 63.51; $\mathrm{H}, 4.40 ; \mathrm{N}$, 12.88; Found: C, 63.59; H, 4.53; N, 12.92.

\subsection{1-(5-(1-(3-Chlorophenyl)-3-(4-methoxyphenyl)-1H-pyrazol-4-yl)-4,5-dihydro-3-}

(thiophen-2yl)pyrazol-1-yl)-ethanone (4)

To a solution of compound $2(0.01 \mathrm{~mol})$ in glacial acetic acid $(20 \mathrm{~mL})$, hydrazine hydrate $(0.01 \mathrm{~mol})$ was added and the mixture was refluxed for $3 \mathrm{~h}$. The reaction mixture was cooled to room temperature and the solid formed was filtered off, dried and recrystallized to get compound 4 in $65 \%$ yield; m.p. 150-154 ${ }^{\circ} \mathrm{C}(\mathrm{EtOH}) ; \mathrm{IR}(\mathrm{KBr})$ v: $3080(\mathrm{CH}-\mathrm{Ar}), 1677(\mathrm{C}=\mathrm{O}), 1619(\mathrm{C}=\mathrm{N}), 1588(\mathrm{C}=\mathrm{C}) \mathrm{cm}^{-1} ;{ }^{1} \mathrm{H}-\mathrm{NMR}$ (DMSO- $\left.d_{6}\right): 2.43\left(\mathrm{~s},{ }^{3} \mathrm{H}, \mathrm{COCH}_{3}\right), 3.30\left(\mathrm{dd},{ }^{1} \mathrm{H}, \mathrm{CH}\right), 3.81\left(\mathrm{~s},{ }^{3} \mathrm{H}, \mathrm{OCH}_{3}\right), 3.88\left(\mathrm{dd},{ }^{1} \mathrm{H}, \mathrm{CH}\right), 5.57\left(\mathrm{dd},{ }^{1} \mathrm{H}\right.$, $\mathrm{CH}), 6.70-7.65\left(\mathrm{~m},{ }^{11} \mathrm{H}, \mathrm{Ar}-\mathrm{H}\right), 9.18\left(\mathrm{~s},{ }^{1} \mathrm{H}, \mathrm{CH}\right.$ of pyrazole) ppm; ${ }^{13} \mathrm{C}-\mathrm{NMR}$ (DMSO- $\left.d_{6}\right): \delta 24.01,43.81$, 55.60, 63.87, 114.26, 116.84, 117.62, 125.35, 125.86, 126.10, 127.56, 129.14, 129.78, 130.69, 131.08, 134.41, 137.82, 140.84, 150.39, 155.19, 159.12, 160.92, 168.19 ppm; MS (EI, $70 \mathrm{eV}): \mathrm{m} / z$ (\%): 476 (43) [M] ${ }^{+}$; Anal. Calcd for $\mathrm{C}_{25} \mathrm{H}_{21} \mathrm{ClN}_{4} \mathrm{O}_{2} \mathrm{~S}$ (476.98): C, 62.95; H, 4.44; N, 11.75; Found: C, 63.02; H, 4.35; N, 11.81 .

\subsection{5-(1-(3-Chlorophenyl)-3-(4-methoxyphenyl)-1H-pyrazol-4-yl)-4,5-dihydro-3-} (thiophen-2-yl)-pyrazole-1-carbothioamide (5)

To a mixture of chalcone $2(0.01 \mathrm{~mol})$ in absolute ethanol $(30 \mathrm{~mL})$, sodium hydroxide $(1 \mathrm{~g}, 0.025 \mathrm{~mol})$ was added. The reaction mixture was heated under reflux for $5 \mathrm{~h}$. The contents were reduced, cooled and poured onto crushed ice. The resulting precipitate was collected by filtration and recrystallized to give in 61\% yield; m.p. $>300{ }^{\circ} \mathrm{C}(\mathrm{MeOH})$; $\mathrm{IR}(\mathrm{KBr})$ v: $3407\left(\mathrm{NH}_{2}\right), 1658(\mathrm{C}=\mathrm{N}), 1523(\mathrm{C}=\mathrm{C}), 1078(\mathrm{C}=\mathrm{S})$ $\mathrm{cm}^{-1} ;{ }^{1} \mathrm{H}-\mathrm{NMR}$ (DMSO- $\left.d_{6}\right): 3.13\left(\mathrm{dd},{ }^{1} \mathrm{H}, \mathrm{CH}\right), 3.84\left(\mathrm{~s},{ }^{3} \mathrm{H}, \mathrm{OCH}_{3}\right), 4.14\left(\mathrm{dd},{ }^{1} \mathrm{H}, \mathrm{CH}\right), 5.51\left(\mathrm{dd},{ }^{1} \mathrm{H}\right.$, $\mathrm{CH}), 7.14-8.02\left(\mathrm{~m},{ }^{11} \mathrm{H}, \mathrm{Ar}-\mathrm{H}\right), 9.12\left(\mathrm{~s},{ }^{1} \mathrm{H}, \mathrm{CH}\right.$ of pyrazole), $9.93\left(\mathrm{~s},{ }^{2} \mathrm{H}, \mathrm{NH}_{2} \mathrm{D}_{2} \mathrm{O}\right.$ exchangeable) ppm; ${ }^{13}$ C-NMR (DMSO- $d_{6}$ ): $\delta 43.14,55.64,62.93,114.33,116.72,117.58,125.37,125.80,126.08,127.54,128.79$, 129.22, 130.65, 131.16, 138.12, 137.82, 140.85, 151.23, 156.19, 159.30, 161.02, 176.55 ppm; MS (EI, 70 eV): 
m/z (\%): 494 (6) [M] $]^{+}$; Anal. Calcd for $\mathrm{C}_{24} \mathrm{H}_{20} \mathrm{ClN}_{5} \mathrm{OS}_{2}$ (494.03): C, 58.35; $\mathrm{H}, 4.08 ; \mathrm{N}, 14.18$; Found: $\mathrm{C}$, $58.27 ; \mathrm{H}, 4.12 ; \mathrm{N}, 14.23$.

3.6. 2-Amino-4-(1-(3-chlorophenyl)-3-(4-methoxyphenyl)-1H-pyrazol-4-yl)-1,6-dihydro-6-oxopyrimidine-5carbonitrile (6a) and 5-acetyl-2-amino-6-(1-(3-chlorophenyl)-3-(4-methoxyphenyl)-1H-pyrazol-4-yl)pyrimidin-4(3H)-one $(\mathbf{6 b})$

A mixture of compound $1(0.01 \mathrm{~mol})$, ethyl cyanoacetate or ethyl acetoacetate $(0.01 \mathrm{~mol})$ and $(5 \mathrm{~mL}$ ) of $40 \%$ ethanolic sodium hydroxide was stirred for $10 \mathrm{~min}$, followed by addition of guanidine hydrochloride $(0.01 \mathrm{~mol})$ and the heating continued under refluxed for $3 \mathrm{~h}$. The reaction mixture was diluted with ice-water and the formed precipitate was collected by filtration, washed several times with water, dried and recrystallized to afford the title compounds $\mathbf{6 a}, \mathbf{b}$.

2-Amino-4-(1-(3-chlorophenyl)-3-(4-methoxyphenyl)-1H-pyrazol-4-yl)-1,6-dihydro-6-oxopyrimidine-5-carbonitrile (6a). Yield 64\%; m.p. 144-148 ${ }^{\circ} \mathrm{C}(\mathrm{EtOH})$; $\mathrm{IR}(\mathrm{KBr})$ v: 3341, $3145\left(\mathrm{NH}_{2}, \mathrm{NH}\right), 2219(\mathrm{C} \equiv \mathrm{N}), 1663(\mathrm{C}=\mathrm{O})$, $1599(\mathrm{C}=\mathrm{C}) \mathrm{cm}^{-1} ;{ }^{1} \mathrm{H}-\mathrm{NMR}$ (DMSO- $\left.d_{6}\right): 2.85\left(\mathrm{~s},{ }^{2} \mathrm{H}, \mathrm{NH}_{2} \mathrm{D}_{2} \mathrm{O}\right.$ exchangeable), $3.88\left(\mathrm{~s},{ }^{3} \mathrm{H}, \mathrm{OCH}_{3}\right)$, 7.03-8.75 (m, ${ }^{9} \mathrm{H}, \mathrm{Ar}-\mathrm{H}$ and $\mathrm{NH} \mathrm{D}_{2} \mathrm{O}$ exchangeable), $9.20\left(\mathrm{~s},{ }^{1} \mathrm{H}, \mathrm{CH}\right.$ of pyrazole) ppm; ${ }^{13} \mathrm{C}-\mathrm{NMR}$ (DMSO- $d_{6}$ ): $\delta 55.63,113.75,115.31,116.79,117.77,123.48,125.21,126.4,127.94,128.30,129.82,130.80$, 134.57, 140.89, 155.04, 159.88, 160.60, 164.33, 171.88 ppm; MS (EI, $70 \mathrm{eV}): \mathrm{m} / z$ (\%): 418 (14) [M] ${ }^{+}$; Anal. Calcd for $\mathrm{C}_{21} \mathrm{H}_{15} \mathrm{ClN}_{6} \mathrm{O}_{2}$ (418.84): C, 60.22; $\mathrm{H}, 3.61 ; \mathrm{N}, 20.07$; Found: $\mathrm{C}, 60.26 ; \mathrm{H}, 3.58 ; \mathrm{N}, 20.15$.

5-Acetyl-2-amino-6-(1-(3-chlorophenyl)-3-(4-methoxyphenyl)-1H-pyrazol-4-yl)pyrimidin-4-(3H)-one (6b). Yield 68\%, m.p. $135-137{ }^{\circ} \mathrm{C}(\mathrm{EtOH})$; IR (KBr) v: 3410, $3152\left(\mathrm{NH}_{2}, \mathrm{NH}\right), 1675(\mathrm{C}=\mathrm{O}), 1589(\mathrm{C}=\mathrm{C})$ $\mathrm{cm}^{-1} ;{ }^{1} \mathrm{H}-\mathrm{NMR}$ (DMSO- $\left.d_{6}\right): 2.30\left(\mathrm{~s},{ }^{2} \mathrm{H}, \mathrm{NH}_{2} \mathrm{D}_{2} \mathrm{O}\right.$ exchangeable), $2.39\left(\mathrm{~s},{ }^{3} \mathrm{H}, \mathrm{CH}_{3}\right), 3.86\left(\mathrm{~s},{ }^{3} \mathrm{H}, \mathrm{OCH}_{3}\right)$, 6.90-7.96 (m, ${ }^{9} \mathrm{H}$, Ar-H and $\mathrm{NH}$ exchangeable with $\left.\mathrm{D}_{2} \mathrm{O}\right), 9.14\left(\mathrm{~s},{ }^{1} \mathrm{H}, \mathrm{CH}\right.$ of pyrazole) ppm; ${ }^{13} \mathrm{C}-\mathrm{NMR}$ (DMSO- $d_{6}$ ): $\delta 24.38,55.60,113.72,115.94,116.85,117.73,125.10,126.01,128.36,130.76,132.95,134.47$, 141.05, 151.14, 154.78, 159.60, 160.82, 164.21, 165.38, 182.70 ppm; MS (EI, 70 eV): $\mathrm{m} / z$ (\%): 435 (7) [M] ; Anal. Calcd for $\mathrm{C}_{22} \mathrm{H}_{18} \mathrm{ClN}_{5} \mathrm{O}_{3}$ (435.86): $\mathrm{C}, 60.62 ; \mathrm{H}, 4.16$; N, 16.07; Found: $\mathrm{C}, 60.59 ; \mathrm{H}, 4.11 ; \mathrm{N}, 16.12$.

\subsection{1-(3-Chlorophenyl)-3-(4-methoxyphenyl)-4-(3-(thiophen-2-yl)isoxazol-5-yl)-1H-pyrazole (7)}

A mixture of compounds $2(0.01 \mathrm{~mol})$ and hydroxylamine hydrochloride $(0.01 \mathrm{~mol})$ in ethanol $(30 \mathrm{~mL})$ containing sodium hydroxide solution $(0.5 \mathrm{~g} \mathrm{NaOH}$ in $0.5 \mathrm{~mL}$ water) was refluxed for $3 \mathrm{~h}$. The reaction mixture was poured onto ice-water, neutralized with drops of conc. Hydrochloric acid and the solid precipitate formed filtered off, washed with water and recrystallized to yield the desired compound 7 in $73 \%$ yield; m.p. $>300{ }^{\circ} \mathrm{C}(\mathrm{EtOH})$; IR (KBr) v: $3064(\mathrm{CH}-\mathrm{Ar}), 1601(\mathrm{C}=\mathrm{C}) \mathrm{cm}^{-1} ;{ }^{1} \mathrm{H}-\mathrm{NMR}$ $\left(\right.$ DMSO- $\left._{6}\right): 3.99\left(\mathrm{~s},{ }^{3} \mathrm{H}, \mathrm{OCH}_{3}\right), 7.30\left(\mathrm{~d},{ }^{2} \mathrm{H}, \mathrm{H} J=20\right), 7.60\left(\mathrm{~d},{ }^{1} \mathrm{H}, \mathrm{CH}\right) ; 7.75-8.45\left(\mathrm{~m},{ }^{10} \mathrm{H}, \mathrm{Ar}-\mathrm{H}\right)$, $9.15\left(\mathrm{~s},{ }^{1} \mathrm{H}, \mathrm{CH}\right.$ of pyrazole) ppm; ${ }^{13} \mathrm{C}-\mathrm{NMR}$ (DMSO- $\left.d_{6}\right): \delta 43.19,55.61,73.10,114.38,116.72,117.68$, 125.68, 125.84, 126.10, 127.43, 129.07, 129.57, 130.81, 131.29, 134.47, 138.21, 140.85, 151.20, 154.63, 160.92, 162.89 ppm; MS (EI, $70 \mathrm{eV}): \mathrm{m} / z$ (\%): 433 (12) [M] ; Anal. Calcd for $\mathrm{C}_{23} \mathrm{H}_{16} \mathrm{ClN}_{3} \mathrm{O}_{2} \mathrm{~S}$ (433.91): C, 63.66; H, 3.72; N, 9.68; Found: C, 63.74; H, 3.79; N, 9.71.

\subsection{4-(1-(3-Chlorophenyl)-3-(4-methoxyphenyl)-1H-pyrazol-4-yl)-6-(thiophen-2-yl)pyrimidin-2-amine (8)}

An aqueous solution $5 \mathrm{~mL}$ of $40 \%$ sodium hydroxide was added gradually during a period of $3 \mathrm{~h}$ to a mixture of chalcone $2(0.01 \mathrm{~mol})$ and guanidine sulfate $(0.01 \mathrm{~mol})$ in ethanol $(25 \mathrm{~mL})$. The reaction mixture was refluxed for $5 \mathrm{~h}$ and the reaction was monitored by TLC. After completion of the reaction, the reaction mixture was poured onto ice-cold water and the solid product formed was collected by filtration, washed with water then recrystallized to get compound 8 in $67 \%$ yield; m.p. $>300{ }^{\circ} \mathrm{C}$ (MeOH); IR (KBr) v: $3347\left(\mathrm{NH}_{2}\right) ; 1632(\mathrm{C}=\mathrm{N}) ; 1589(\mathrm{C}=\mathrm{C}) \mathrm{cm}^{-1} ;{ }^{1} \mathrm{H}-\mathrm{NMR}$ (DMSO- $\left.d_{6}\right): 3.80\left(\mathrm{~s},{ }^{3} \mathrm{H}\right.$, $\left.\mathrm{OCH}_{3}\right), 6.68-8.08\left(\mathrm{t},{ }^{12} \mathrm{H}, \mathrm{Ar}-\mathrm{H}\right), 8.93\left(\mathrm{~s},{ }^{1} \mathrm{H}, \mathrm{CH}\right.$ of pyrazole $), 10.18\left(\mathrm{~s},{ }^{2} \mathrm{H}, \mathrm{NH}_{2} \mathrm{D}_{2} \mathrm{O}\right.$ exchangeable) ppm; ${ }^{13}$ C-NMR (DMSO- $d_{6}$ ): $\delta 55.64,82.10,114.31,116.80,117.59,125.30,125.78,126.09,127.41,128.67,129.48$, 130.79, 131.16, 134.49, 139.86, 141.05, 150.88, 152.10, 160.37, 164.21, 166.58, 164.25 ppm; Anal. Calcd for $\mathrm{C}_{24} \mathrm{H}_{18} \mathrm{ClN}_{5} \mathrm{OS}$ (459.95): C, 62.67; H, 3.94; N, 15.23; Found: C, 62.63; H, 3.88; N, 15.29. 


\subsection{6-(1-(3-Chlorophenyl)-3-(4-methoxyphenyl)-1H-pyrazol-4-yl)-4-(thiophen-2-yl)pyrimidine-2-(1H)-thione (9)}

A solution of the chalcone $2(0.01 \mathrm{~mol})$, thiourea $(0.01 \mathrm{~mol})$ and sodium hydroxide $(0.1 \mathrm{~g})$ in absolute ethanol $(30 \mathrm{~mL})$ was refluxed for $6 \mathrm{~h}$. The reaction mixture was concentrated under vacuum, cooled and neutralized with dilute $\mathrm{HCl}$. The formed product was filtered off, washed with water and recrystallized to get compound 9 in $76 \%$ yield; m.p. $>300{ }^{\circ} \mathrm{C}(\mathrm{MeOH}) ; \mathrm{IR}(\mathrm{KBr})$ v: $3422(\mathrm{NH})$, $1595(\mathrm{C}=\mathrm{C}), 1176(\mathrm{C}=\mathrm{S}) \mathrm{cm}^{-1},{ }^{1} \mathrm{H}-\mathrm{NMR}\left(\mathrm{DMSO}-d_{6}\right): 3.82\left(\mathrm{~s},{ }^{3} \mathrm{H}, \mathrm{OCH}_{3}\right), 6.90-8.60\left(\mathrm{~m},{ }^{12} \mathrm{H}, \mathrm{Ar}-\mathrm{H}\right)$, $9.20\left(\mathrm{~s},{ }^{1} \mathrm{H}, \mathrm{CH}\right.$ of pyrazole), $9.95\left(\mathrm{~s},{ }^{1} \mathrm{H}, \mathrm{NH} \mathrm{D} 2 \mathrm{O}\right.$ exchangeable) ppm; ${ }^{13} \mathrm{C}-\mathrm{NMR}$ (DMSO- $\left.d_{6}\right): \delta 55.63$, 104.56, 113.82, 115.89, 117.66, 125.38, 125.70, 126.03, 127.38, 128.34, 129.45, 130.77, 131.09, 134.42, 138.17, 140.95, 150.76, 157.21, 160.85, 161.80, 162.46, 184.33 ppm; MS (EI, $70 \mathrm{eV}): 477$ (8) [M] ${ }^{+}$; Anal. Calcd for $\mathrm{C}_{24} \mathrm{H}_{17} \mathrm{ClN}_{4} \mathrm{OS}_{2}$ (477): C, 60.43; H, 3.59; N, 11.75; Found: C, 60.38; H, 3.66; N, 11.82.

\subsection{Measurement of Anticancer Activity}

The experimental method used in anticancer screening has been adopted by U.S. National Cancer Institute according to reported standard procedure [28-30].

\section{Conclusions}

In summary, we have synthesized a series of novel pyrazole derivatives incorporated different heteroaryl ring systems in one molecule and evaluated these compounds for their anticancer activities against different 60 human cancer cell lines representing leukemia, melanoma and cancers of lung, colon, brain, ovary, breast, prostate and kidney cancer using a two-stage process. The pyrimidine-2(1H)-thione derivative 9 showed good anticancer activity with $\left(\mathrm{GI}_{50}\right.$ MG-MID $=3.59 \mu \mathrm{M}$ ) compared to the standard drug sorafenib. The structures of the new compounds were elucidated using spectroscopic and elemental analysis.

Acknowledgments: The project was financially supported by King Saud University, Vice Deanship of Research Chairs.

Author Contributions: The listed authors contributed to this work as described in the following. Hoda H. Fahmy gave the concepts of the work, interpreted the results and prepared the manuscript, Eman S. Nossier, carried out the synthetic work, interpreted the results and prepared the manuscript and Nagy M. Khalifa, Magda M. F. Ismail and Hend M. El-Sahrawy interpreted the results and cooperated in the preparation of the manuscript. All authors read and approved the final manuscript.

Conflicts of Interest: The authors declare no conflict of interest.

\section{References}

1. Jemal, A.; Siegel, R.; Ward, E.; Hao, Y.; Xu, J.; Murray, T.; Thun, M.J. Cancer statistics, 2008. Cancer J. Clin. 2008, 58, 71-96. [CrossRef] [PubMed]

2. Eckhardt, S. Recent progress in the development of anticancer agents. Curr. Med. Chem. Anticancer Agents 2002, 2, 419-439. [CrossRef] [PubMed]

3. Altmann, K.H. Microtubule-stabilizing agents: A growing class of important anticancer drugs. Curr. Opin. Chem. Biol. 2001, 5, 424-431. [CrossRef]

4. Wartmann, M.; Altmann, K.H. The biology and medicinal chemistry of epothilones. Curr. Med. Chem. Anticancer Agents 2002, 2, 1231-1248. [CrossRef]

5. O'Dwyer, M.E.; Druker, B.J. The role of the tyrosine kinase inhibitor STI571 in the treatment of cancer. Curr. Cancer Drug Targets 2001, 1, 49-57. [CrossRef] [PubMed]

6. Xia, Y.; Dong, Z.W.; Zhao, B.X.; Ge, X.; Meng, N.; Shin, D.S.; Miao, J.Y. Synthesis and structure-activity relationships of novel 1-arylmethyl-3-aryl-1H-pyrazole-5-carbohydrazide derivatives as potential agents against A549 lung cancer cells. Bioorg. Med. Chem. 2007, 15, 6893-6899. [CrossRef] [PubMed]

7. Farghaly, A.R. Synthesis of some new indole derivatives containing pyrazoles with potential antitumor activity. ARKIVOC 2010, 11, 177-187. 
8. El-Zahar, M.I.; EL-Karim, S.A.; Haiba, M.E.; Khedr, M.A. Synthesis, antitumor activity and molecular docking study of novel benzofuran-2-yl pyrazole pyrimidine derivatives. Acta Pol. Pharm. Drug. Res. 2011, 68, 357-373.

9. Kalirajan, R.; Rathore, L.; Jubie, S.; Gowramma, B.; Gomathy, S.; Sankar, S. Microwave assisted synthesis of some novel pyrazole substituted benzimidazoles and evaluation of their biological activities. Indian J. Chem. 2011, 50B, 1794-1799. [CrossRef]

10. Perchellet, E.M.; Ward, M.M.; Skaltsounis, A.L.; Kostakis, I.K.; Pouli, N.; Marakos, P.; Perchellet, J.H. Antiproliferative and proapoptotic activities of pyranoxanthenones, pyranothioxanthenones and their pyrazole-fused derivatives in HL-60 cells. Anticancer Res. 2006, 26, 2791-2804. [PubMed]

11. Insuasty, B.; Tigreros, A.; Orozco, F.; Quiroga, J.; Abonia, R.; Nogueras, M.; Sanchez, A.; Cobo, J. Synthesis of novel pyrazolic analogues of chalcones and their 3-aryl-4-(3-aryl-4,5-dihydro-1H-pyrazol-5-yl)-1-phenyl$1 H$-pyrazole derivatives as potential antitumor agents. Bioorg. Med. Chem. 2010, 18, 4965-4974. [CrossRef] [PubMed]

12. Labbozzetta, M.; Baruchello, R.; Marchetti, P.; Gueli, M.C.; Poma, P.; Notarbartolo, M.; Simoni, D.; D'Alessandro, N. Lack of nucleophilic addition in the isoxazole and pyrazole diketone modified analogs of curcumin; implications for their antitumor and chemosensitizing activities. Chem. Biol. Interact. 2009, 181, 29-36. [CrossRef] [PubMed]

13. Szabo, G.; Fischer, J.; Kis-Varga, A.; Gyires, K. New celecoxib derivatives as anti-inflammatory agents. J. Med. Chem. 2008, 51, 142-147. [CrossRef] [PubMed]

14. Tanitame, A.; Oyamada, Y.; Ofuji, K.; Terauchi, H.; Kawasaki, M.; Wachi, M.; Yamagishi, J. Synthesis and antibacterial activity of a novel series of DNA gyrase inhibitors: 5-[(E)-2-arylvinyl]pyrazoles. Bioorg. Med. Chem. Lett. 2005, 15, 4299-4303. [CrossRef] [PubMed]

15. Rida, S.M.; Saudi, M.N.S.; Youssef, A.M.; Halim, M.A. Synthesis and biological evaluation of the pyrazole class of cyclooxygenase-2-inhibitors. Lett. Org. Chem. 2009, 6, 282-288. [CrossRef]

16. Abadi, A.H.; Abdel Haleem, A.; Hassan, G.S. Synthesis of novel 1,3,4-trisubstituted pyrazole derivatives and their evaluation as antitumor and antiangiogenic agents. Chem. Pharm. Bul. 2003, 51, 838-844. [CrossRef]

17. Sridhar, S.; Parsad, R.Y. Synthesis and Analgesic Studies of Some New 2-pyrazolines. Eur. J. Chem. 2012, 9, 1810-1815. [CrossRef]

18. Turan-Zitouni, G.; Chevallet, P.; Kilic, F.S.; Erol, K. Synthesis of some thiazolyl-pyrazoline derivatives and preliminary investigation of their hypotensive activity. Eur. J. Med. Chem. 2000, 35, 635-641. [CrossRef]

19. Zhou, S.; Ren, J.; Liu, M.; Ren, L.; Liu, Y.; Gong, P. Design, synthesis and pharmacological evaluation of 6,7-disubstituted-4-phenoxyquinoline derivatives as potential antitumor agents. Bioorg. Chem. 2014, 57, 30-42. [CrossRef] [PubMed]

20. Kumar, S.; Bawa, S.; Drabu, S.; Kumar, R.; Gupta, H. Biological activities of pyrazoline derivatives-A recent development. Drug Discov. 2009, 4, 154-163. [CrossRef]

21. Havrylyuk, D.; Zimenkovsky, B.; Vasylenko, O.; Zaprutko, L.; Gzella, A.; Lesyk, R. Synthesis of novel thiazolone-based compounds containing pyrazoline moiety and evaluation of their anticancer activity. Eur. J. Med. Chem. 2009, 44, 1396-1404. [CrossRef] [PubMed]

22. Khalifa, N.M.; Al-Omar, M.A.; Amr, A.E.; Baiuomy, A.R.; Abdel Rahman, R.F. Synthesis and biological evaluation of some novel fused thiazolo[3,2-a]pyrimidines as potential analgesic and antiinflammatory agents. Russ. J. Bioorg. Chem. 2015, 41, 192-201. [CrossRef]

23. Eweas, A.F.; Khalifa, N.M.; Ismail, N.S.; Al-Omar, M.A.; Soliman, A.M. Synthesis, molecular docking of novel 1,8-naphthyridine derivatives and their cytotoxic activity against HepG2 cell lines. Med Chem Res. 2014, 23, 76-86. [CrossRef]

24. Almutairi, M.S.; Hegazy, G.H.; Haiba, M.E.; Ali, H.I.; Khalifa, N.M.; Soliman, A.M. Synthesis, Docking and Biological Activities of Novel Hybrids Celecoxib and Anthraquinone Analogs as Potent Cytotoxic Agents. Int. J. Mol. Sci. 2014, 15, 22580-22603. [CrossRef] [PubMed]

25. Haiba, M.E.; Al-Abdullah, E.S.; Edrees, M.M.; Khalifa, N.M. Synthesis and characterization of some substituted 3,4-dihydronaphthalene derivatives through different enaminones as potent cytotoxic agents. Drug Res. 2015, 65, 9-17.

26. Babasaheb, P.B.; Shrikant, S.G.; Ragini, G.B.; Jalinder, V.T.; Chandrahas, N.K. Synthesis and biological evaluation of simple methoxylated chalcones as anticancer, anti-inflammatory and antioxidant agents. Bioorg. Med. Chem. 2010, 18, 1364-1370. 
27. Syam, S.; Abdelwahab, S.I.; Al-Mamary, M.A.; Mohan, S. Synthesis of chalcones with anticancer activities. Molecules 2012, 17, 6179-6195. [CrossRef] [PubMed]

28. Alley, M.C.; Scudiero, D.A.; Monks, P.A.; Hursey, M.L.; Fine, M.J.; Czerwinski, D.L.; Abbott, B.J.; Mayo, J.G.; Shoemaker, R.H.; Boyd, M.R. Feasibility of drug screening with panels of human tumor cell lines using a microculture tetrazolium assay. Cancer Res. 1988, 48, 589-601. [PubMed]

29. Grever, M.R.; Schepartz, S.A.; Chabner, B.A. The National Cancer Institute: Cancer drug discovery and development program. Semin. Oncol. 1992, 19, 622-638. [PubMed]

30. Boyd, M.R.; Paull, K.D. Some practical considerations and applications of the National Cancer Institute in vitro anticancer drug discovery screen. Drug Dev. Res. 1995, 34, 91-109. [CrossRef]

Sample Availability: Samples of all the compounds are available from the authors.

(C) 2016 by the authors; licensee MDPI, Basel, Switzerland. This article is an open access article distributed under the terms and conditions of the Creative Commons by Attribution (CC-BY) license (http:/ / creativecommons.org/licenses/by/4.0/). 